\title{
New potential diagnostic biomarkers for pulmonary hypertension
}

\author{
Svenja L. Tiede ${ }^{1,8}$, Henning Gall ${ }^{1,2,8}$, Oliver Dörr ${ }^{3}$, Marina dos Santos Guilherme ${ }^{1,3}$, \\ Christian Troidl $^{4}$, Christoph Liebetrau ${ }^{4}$, Sandra Voss ${ }^{4}$, Robert Voswinckel ${ }^{1}$, \\ Ralph T. Schermuly ${ }^{1}$, Werner Seeger ${ }^{1}$, Friedrich Grimminger ${ }^{1,5}$, \\ Andreas M. Zeiher ${ }^{4}$, Stefanie Dimmeler ${ }^{6}$, Helge Möllmann ${ }^{6}$, Christian W. Hamm ${ }^{6}$, \\ Hossein Ardeschir Ghofrani ${ }^{1,5,7}$ and Holger M. Nef ${ }^{3}$
}

Affiliations: ${ }^{1}$ Universities of Giessen and Marburg Lung Center (UGMLC), member of the German Center for Lung Research (DZL), Giessen, Germany. ${ }^{2}$ Dept of Epidemiology, Erasmus MC, Rotterdam, The Netherlands. ${ }^{3}$ Dept of Internal Medicine I, Cardiology/Angiology, Giessen University, Giessen, Germany. 'Dept of Cardiology, Kerckhoff Heart Center, Bad Nauheim, Germany. ${ }^{5}$ Dept of Pneumology, Kerckhoff Clinic, Bad Nauheim, Germany. ${ }^{6}$ Department of Cardiology and Molecular Cardiology, Johann Wolfgang Goethe University, Frankfurt, Germany. ${ }^{7}$ Dept of Medicine, Imperial College London, London, UK. ${ }^{8}$ Both authors contributed equally to this work.

Correspondence: Ardeschir Ghofrani, Pulmonary Hypertension Division, Medical Clinic II/V, University Hospital Giessen und Marburg GmbH, Klinikstrasse 36, 35392 Giessen, Germany.

E-mail: ardeschir.ghofranidinnere.med.uni-giessen.de

ABSTRACT This study aimed to determine whether the vascular endothelial growth factor (VEGF) family members soluble VEGF receptor 1 (also called soluble fms-like tyrosine kinase 1 (sFlt-1)) and placental growth factor (PlGF) could be used as biomarkers for pulmonary hypertension $(\mathrm{PH})$.

Consecutive patients undergoing right heart catheterisation were enrolled (those with mean pulmonary arterial pressure $\geqslant 25 \mathrm{mmHg}$ were classed as having $\mathrm{PH}$; those with mean pulmonary arterial pressure $<25 \mathrm{mmHg}$ acted as non-PH controls). Plasma from the time of PH diagnosis was analysed for PlGF and sFlt-1 using enzyme immunoassays.

In total, 247 patients with $\mathrm{PH}$ were enrolled: 62 with idiopathic pulmonary arterial hypertension (IPAH), 14 with associated pulmonary arterial hypertension (APAH), 21 with collagen vascular disease (CVD), 26 with pulmonary venous hypertension, 67 with lung disease-associated $\mathrm{PH}$ and 57 with chronic thromboembolic PH. The non-PH control group consisted of 40 patients. sFlt-1 plasma levels were significantly higher in patients with IPAH, APAH, CVD and lung disease-associated PH versus controls; PIGF levels were significantly higher in all PH groups versus controls. The combination of sFlt-1 and PlGF resulted in a sensitivity of $83.7 \%$ with specificity of $100 \%$ for pulmonary arterial hypertension. There was no association between sFlt-1 or PlGF and haemodynamic parameters, 6-min walking distance or survival.

In summary, $\mathrm{PlGF}$ and sFlt-1 are promising diagnostic biomarkers for $\mathrm{PH}$.

@ERSpublications

This study shows a high potential for VEGF family members as diagnostic biomarkers for pulmonary hypertension http://ow.ly/P5GCW

This article has been revised according to the correction published in the September 2016 issue of the European Respiratory Journal.

This article has supplementary material available from erj.ersjournals.com

Received: Feb 022015 | Accepted after revision: June 082015 | First published online: Aug 062015

Conflict of interest: Disclosures can be found alongside the online version of this article at erj.ersjournals.com

Copyright OERS 2015 


\section{Introduction}

Pulmonary hypertension $(\mathrm{PH})$ is defined as resting mean pulmonary arterial pressure (mean PAP) $\geqslant 25 \mathrm{mmHg}$ [1]. Different $\mathrm{PH}$ aetiologies have resulted in a classification of $\mathrm{PH}$ into five groups, but regardless of aetiology, $\mathrm{PH}$ involves vasoconstriction, media hypertrophy, and in situ thrombosis, leading to an increase in pulmonary arterial pressure, ultimately resulting in right heart failure [2].

Early diagnosis and monitoring of disease progression are critical for therapy decisions. Right heart catheterisation remains the gold standard for diagnosing $\mathrm{PH}$ and managing those patients who are receiving $\mathrm{PH}$ therapy $[1,3]$. Brain natriuretic peptide (BNP) is an established prognostic biomarker to monitor right heart failure in $\mathrm{PH}$ [4], and reflects myocardial stress. There is, nevertheless, still a need for other non-invasive $\mathrm{PH}$ biomarkers that mirror pathological alterations that occur in the pulmonary vasculature and which also help to diagnose $\mathrm{PH}$.

Vascular endothelial growth factor (VEGF) signalling is known to be associated with PH pathogenesis, particularly in vascular remodelling [5]. VEGF expression can be induced by hypoxia through upregulation of hypoxia-inducible factor-1 $\alpha$ (HIF-1 $\alpha$ ) [6]. Moreover, VEGF and VEGF receptor 2 (VEGFR-2) are overexpressed in plexiform lesions of patients with PH [7] and plasma VEGF levels are elevated in patients with idiopathic pulmonary arterial hypertension (IPAH) [8].

VEGF receptors are membrane-bound receptor tyrosine kinases. The soluble form of the VEGF receptor 1 (also called soluble fms-like tyrosine kinase 1 (sFlt-1)) results from alternative splicing or cleavage of the full-length receptor, Flt-1 [9]. The VEGF family member placental growth factor (PlGF) binds exclusively to Flt-1 [10], which leads to pro-angiogenic signalling through several mechanisms, including direct intracellular activation of Flt-1 and downstream target genes, and transphosphorylation of VEGFR-2 by activated Flt-1, thereby increasing the response to VEGF [11]. Moreover, the PlGF/VEGF-A heterodimer can bind and activate Flt-1 and induce Flt-1/VEGFR-2 dimerisation [12].

PlGF and sFlt-1 have shown diagnostic and prognostic potential in hypoxia-associated preeclampsia [13, 14], in sickle cell disease-associated $\mathrm{PH}[8,15]$ and in peripheral and coronary artery disease [16]. In a recent study, MaLHOTRA et al. [17] showed significantly upregulated sFlt-1 serum levels in pulmonary arterial hypertension (PAH) patients. Serum sFlt-1 levels were associated with increased New York Heart Association (NYHA) functional class and predict survival.

We hypothesise that Flt-1 and PlGF represent important factors in $\mathrm{PH}$ and can be used as biomarkers.

\section{Methods}

\section{Patients and biomarker study design}

Plasma levels of PlGF and sFlt-1 were measured in patients with five different subtypes of $\mathrm{PH}$ and compared with a non-PH control group, all undergoing right heart catheterisation. Furthermore, plasma levels were correlated with haemodynamics, NYHA functional class and survival.

For this case-control study, consecutive patients with suspicion of $\mathrm{PH}$, undergoing right heart catheterisation at the Giessen PH referral centre (Germany), were enrolled in the study. Blood samples were taken at the baseline visit during right heart catheterisation. The baseline visit was the first right heart catheter and the diagnosis or exclusion of $\mathrm{PH}$ for all patients. Patients with mean PAP $\geqslant 25 \mathrm{mmHg}$ were defined as having PH. Patients undergoing right heart catheterisation with mean PAP $<25 \mathrm{mmHg}$ were defined as non-PH controls. The non- $\mathrm{PH}$ controls underwent right heart catheterisation because symptoms led to suspicion of $\mathrm{PH}$, which was excluded by right heart catheterisation. $\mathrm{PH}$ Patients were classified into aetiological groups according to current guidelines [3]. Plasma was collected using EDTA as an anticoagulant followed by centrifugation at $3000 \times \mathrm{g}$ for $10 \mathrm{~min}$ and samples were then frozen at $-80^{\circ} \mathrm{C}$ until analysis. The approval of the Local Research Ethics Committee at the University Hospital of Giessen was obtained. All patients gave written, informed consent for use and storage of plasma and future biomarker analyses on the day the samples were obtained.

\section{Biomarker measurements}

PlGF and sFlt-1 were measured with commercially available enzyme immunoassays (Quantikine, R\&D Systems, Minneapolis, MN, USA). Samples were assayed in duplicate according to manufacturer's instructions.

\section{Statistical analysis}

Baseline characteristics are presented as mean $\pm \mathrm{SD}$ or median (interquartile range) where appropriate. Kaplan-Meier curves were constructed and log-rank tests performed to compare survival distributions. The association of biomarker concentrations with survival was tested using univariate and multivariate

Cox regression analyses. PlGF and sFlt-1 plasma levels were expressed as mean \pm sEm. One-way ANOVA, 
Tukey post hoc test, Chi-squared test, or Kruskal-Wallis test were used for comparisons between groups, as appropriate. In order to evaluate the performance of sFlt-1 and PlGF as predictors for mortality rates, the area under the curve (AUC) of the receiver operating characteristics (ROC) curve was calculated. Correlation analyses were done with Pearson's or Spearman's co-efficient, as appropriate. A p-value $<0.05$ was considered statistically significant. Statistical analyses were performed using IBM SPSS Statistics 21.0 (IBM, Armonk, NY, USA).

\section{Results}

\section{Demographic characteristics}

The demographic characteristics of all patients enrolled in the main biomarker study are shown in table 1. In total, 247 patients with PH were enrolled: 62 patients with IPAH, 14 with associated PAH (APAH, i.e. Eisenmenger's syndrome, HIV, portopulmonary hypertension), 21 with collagen vascular disease (CVD), 26 with pulmonary venous hypertension $(\mathrm{PVH}), 67$ with lung disease associated pulmonary hypertension (LD-PH) and 57 with chronic thromboembolic PH (CTEPH). Non-PH controls consisted of 40 patients with invasive exclusion of $\mathrm{PH}$ due to similar symptoms as $\mathrm{PH}$ patients.

\section{Circulating sFlt-1 and PIGF in patients with PH}

Blood samples were taken from all patients at the baseline visit, and sFlt-1 and PlGF levels measured. Figure 1 shows sFlt-1 (figure 1a) and PlGF (figure 1b) plasma levels from patients with $\mathrm{PH}$ and the non-PH control group. Mean levels of sFlt-1 were markedly elevated in all PAH subgroups (IPAH, APAH and CVD). Furthermore, sFlt-1 was also significantly elevated in patients with LD-PH. No statistically significant difference was detected between either the CTEPH or the PVH groups and the non-PH control group. Actual mean plasma sFlt-1 concentrations and p-values for $\mathrm{PH}$ groups in comparison with the non-PH control group (mean sFlt-1 plasma concentration $3091.6 \pm 246.5 \mathrm{pg} \cdot \mathrm{mL}^{-1}$ ) were as follows: IPAH $5049.2 \pm 460.3 \mathrm{pg} \cdot \mathrm{mL}^{-1}(\mathrm{p}=0.045) ;$ APAH $6906.1 \pm 1022.1 \mathrm{pg} \cdot \mathrm{mL}^{-1}(\mathrm{p}=0.003) ; \mathrm{CVD}^{2} 7174.4 \pm 1056.1 \mathrm{pg} \cdot \mathrm{mL}^{-1}$ $(\mathrm{p}<0.001)$; PVH $3945.1 \pm 628.9 \mathrm{pg} \cdot \mathrm{mL}^{-1}(\mathrm{p}=0.940)$; LD-PH $5338.2 \pm 390 \mathrm{pg} \cdot \mathrm{mL}^{-1} \quad(\mathrm{p}=0.009)$; and CTEPH $3701.4 \pm 353 \mathrm{pg} \cdot \mathrm{mL}^{-1}(\mathrm{p}=0.969)$. sFlt-1 plasma levels were significantly higher in patients with APAH $(\mathrm{p}=0.016)$ or CVD $(\mathrm{p}=0.001)$ compared with CTEPH patients, as well as in patients with CVD versus those with PVH $(\mathrm{p}=0.012)$.

PlGF levels were elevated significantly in all PH subgroups compared with the non-PH control group (fig. 1b). Actual mean plasma PIGF concentrations and $\mathrm{p}$-values for $\mathrm{PH}$ groups in comparison with the non- $\mathrm{PH}$ control group (mean PlGF plasma concentration $21.8 \pm 1.5 \mathrm{pg} \cdot \mathrm{mL}^{-1}$ ) were as follows: IPAH $45.6 \pm 3.6 \mathrm{pg} \cdot \mathrm{mL}^{-1}$ $(\mathrm{p}<0.001) ;$ APAH $54.3 \pm 9.1 \mathrm{pg} \cdot \mathrm{mL}^{-1}(\mathrm{p}<0.001) ; \mathrm{CVD} 56.3 \pm 4 \mathrm{pg} \cdot \mathrm{mL}^{-1} \quad(\mathrm{p}<0.001) ; \mathrm{PVH} 40 \pm 3.7 \mathrm{pg} \cdot \mathrm{mL}^{-1}$ $(\mathrm{p}=0.030)$; LD-PH 53.4 $\pm 3.4 \mathrm{pg} \cdot \mathrm{mL}^{-1}(\mathrm{p}<0.001)$; and CTEPH $39.3 \pm 3.1 \mathrm{pg} \cdot \mathrm{mL}^{-1}(\mathrm{p}=0.005)$.

TABLE 1 Demographics for enrolled patients with a diagnosis of pulmonary hypertension

\begin{tabular}{|c|c|c|c|c|c|c|}
\hline & IPAH & APAH & CVD & PVH & LD-PH & СTEPH \\
\hline Patients $\mathrm{n}$ & 62 & 14 & 21 & 26 & 67 & 57 \\
\hline Age years & $48.6 \pm 16.1$ & $50.4 \pm 11$ & $58.7 \pm 14.2$ & $64.3 \pm 14$ & $65.6 \pm 9.3$ & $64.6 \pm 14$ \\
\hline \multicolumn{7}{|c|}{ NYHA functional class \% } \\
\hline II & 11.7 & 28.6 & 14.3 & 15.4 & 4.5 & 12.1 \\
\hline III & 71.7 & 42.9 & 33.3 & 61.5 & 56.7 & 60.3 \\
\hline BNP $\mathrm{pg} \cdot \mathrm{mL}^{-1}$ & 102.5 (220.5) & $99(162)$ & 131 (250.5) & 150 (137.8) & 92 (279) & $116.5(306)$ \\
\hline Creatinine $\mathrm{mg} \cdot \mathrm{dL}^{-1}$ & $1.2 \pm 0.3$ & $1.2 \pm 0.2$ & $1.4 \pm 0.7$ & $1.3 \pm 0.5$ & $1.2 \pm 0.3$ & $1.3 \pm 0.4$ \\
\hline Mean PAP mmHg & $56.6 \pm 17.6$ & $50.7 \pm 14.3$ & $43 \pm 10.3$ & $40.7 \pm 15.1$ & $36.8 \pm 13.4$ & $42.7 \pm 14.4$ \\
\hline PCWP mmHg & $7.8 \pm 2.9$ & $7.9 \pm 2.9$ & $8.3 \pm 3.5$ & $19.5 \pm 7.7$ & $8.0 \pm 3.3$ & $9.8 \pm 4.5$ \\
\hline $\mathrm{CI} \mathrm{L} \cdot \mathrm{min}^{-1} \cdot \mathrm{m}^{-2}$ & $2.2 \pm 0.6$ & $2.7 \pm 1.0$ & $2.3 \pm 0.8$ & $2.6 \pm 0.6$ & $2.4 \pm 0.6$ & $2.2 \pm 0.6$ \\
\hline PVR dyn $\cdot \mathrm{S} \cdot \mathrm{cm}^{-5}$ & 939 (1865) & $650(904)$ & $677(614)$ & $282(257)$ & 478 (402) & $626(606)$ \\
\hline
\end{tabular}

Data are presented as mean \pm SD or median (interquartile range), unless otherwise stated. IPAH: idiopathic pulmonary arterial hypertension; APAH: associated pulmonary arterial hypertension; CVD: collagen vascular disease; PVH: pulmonary venous hypertension; LD-PH: lung disease associated pulmonary hypertension; CTEPH: chronic thromboembolic pulmonary hypertension; NYHA: New York Heart Association; 6MWD: 6-min walking distance; BNP: brain natriuretic peptide; mean PAP: mean pulmonary arterial pressure; PCWP: pulmonary capillary wedge pressure; $\mathrm{Cl}$ : cardiac index; PVR: pulmonary vascular resistance; $\mathrm{SvO}_{2}$ : mixed venous oxygen saturation. 

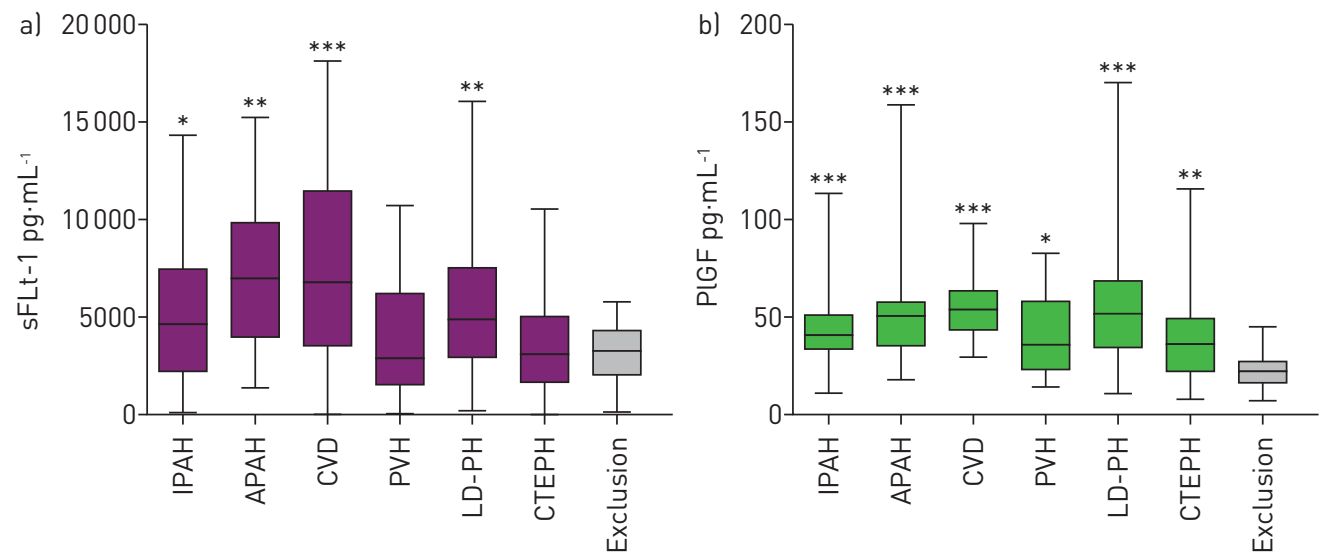

FIGURE 1 Levels of a) soluble fms-like tyrosine kinase 1 (sFlt-1) and b) placental growth factor (PIGF) in patients with pulmonary hypertension $(\mathrm{PH})$ and controls without $\mathrm{PH}$ (exclusion), expressed as mean \pm SEM. Results for all $\mathrm{PH}$ groups were compared with the control group (exclusion) using one-way ANOVA with Tukey's post hoc test. IPAH: idiopathic pulmonary arterial hypertension; APAH: associated pulmonary arterial hypertension; CVD: collagen vascular disease; $\mathrm{PVH}$ : pulmonary venous hypertension; LD-PH: lung disease associated pulmonary hypertension; CTEPH: chronic thromboembolic pulmonary hypertension. *: $p<0.05$; **: $p<0.01 ;{ }^{* * *}: p<0.001$

No significant differences in sFlt-1 and PlGF levels were detected between men and women. Mean plasma concentrations across all PH groups for men and women were as follows: sFlt-1 5071 and $4916 \mathrm{pg} \cdot \mathrm{mL}^{-1}$, respectively ( $\mathrm{p}=0.733)$; and PlGF 48.6 and $45.8 \mathrm{pg} \cdot \mathrm{mL}^{-1}$, respectively $(\mathrm{p}=0.393)$. Mean plasma concentrations across PAH groups (i.e. IPAH, APAH and CVD) for men and women were as follows: sFlt-1 6389 and $5491 \mathrm{pg} \cdot \mathrm{mL}^{-1}$, respectively $(\mathrm{p}=0.307)$; and PlGF 53.3 and $48.1 \mathrm{pg} \cdot \mathrm{mL}^{-1}$, respectively $(\mathrm{p}=0.376)$.

\section{sFlt-1 and PIGF as diagnostic markers for PH}

ROC analysis was performed and the best cut-off values chosen for sFlt-1 (5753 pg.mL $\mathrm{m}^{-1}$ ) with an AUC of 0.662 (95\% CI 0.592-0.733, p=0.001) and for PlGF (29.2 pg. $\mathrm{mL}^{-1}$ ) with an AUC of 0.847 (95\% CI 0.797$0.898, \mathrm{p}<0.001)$. Specificity was $100 \%$ for sFlt- 1 and $85 \%$ for PlGF when using the aforementioned cut-off-values. Likewise, sensitivity values were $36 \%$ for sFlt-1 and $77 \%$ for PlGF. The combination of sFlt-1 and PlGF resulted in a sensitivity of $62.4 \%$ and specificity of $100 \%$ (Chi-squared $\mathrm{p}<0.001$ for both). For combination analysis, only patients with both biomarker values above or both below the cut-off were included.

If ROC analysis were performed only for the PAH group (i.e. IPAH, APAH and CVD), the best cut-off value for sFlt-1 was $5753 \mathrm{pg} \cdot \mathrm{mL}^{-1}$ with an AUC of 0.765 (95\% CI 0.681-0.848; $\mathrm{p}<0.001$; figure 2) and the best cut-off value for PlGF was $29.2 \mathrm{pg} \cdot \mathrm{mL}^{-1}$ with an AUC of 0.905 (95\% CI 0.849-0.960; $<<0.001$; figure 2). For the PAH group, the specificity was $100 \%$ for sFlt -1 and $85 \%$ for PlGF, whilst the sensitivity was $43.3 \%$ for sFlt- 1 and $83.7 \%$ for PlGF. The combination of sFlt-1 and PlGF resulted in a sensitivity of $83.7 \%$ with specificity of $100 \%$ (Chi-squared $\mathrm{p}<0.001$ for both).

BNP was added to the analysis for diagnostic accuracy. ROC analysis reveals an AUC of 0.574 with $\mathrm{p}=0.18$ for BNP alone, and an AUC of 0.577 with $\mathrm{p}=0.16$ when combined with PlGF and sFlt- 1.

FIGURE 2 Soluble fms-like tyrosine kinase 1 (sFlt-1) and placental growth factor (PIGF) as diagnostic markers for all pulmonary arterial hypertension aetiological groups. Receiver operating characteristic curves of sFlt-1 (blue) and PIGF (green) to predict a diagnosis of pulmonary hypertension. Area under the receiver operating characteristic curve for sFlt- 1 was $0.765195 \% \mathrm{Cl}$ $0.681-0.848 ; p<0.001)$ and for PIGF was $0.905 \quad 95 \% \mathrm{Cl} \quad 0.849-0.960$; $\mathrm{p}<0.0011$.

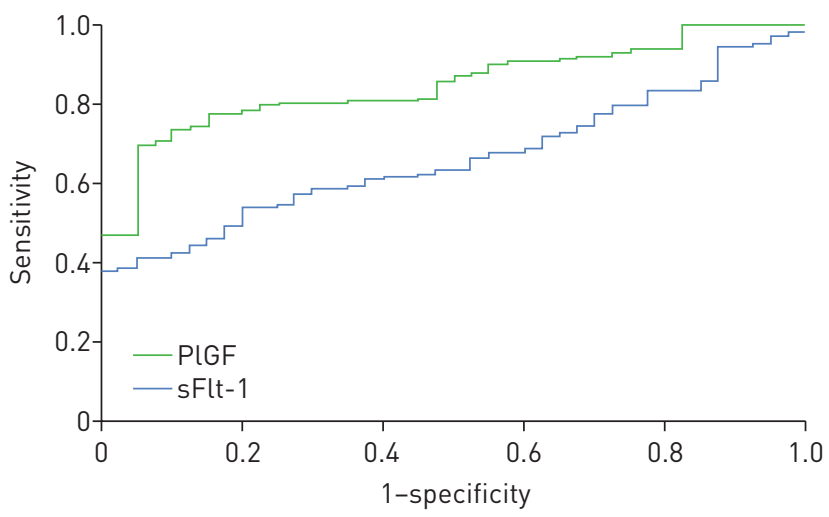


sFlt-1 and PIGF to estimate severity of disease

Neither sFlt-1 nor PlGF correlated with mean PAP, pulmonary vascular resistance (PVR), 6-min walking distance (6MWD) or BNP. Across all PH aetiologies the plasma concentration of sFlt-1 was significantly different between NYHA classes ( $\mathrm{p}=0.015$, Kruskal-Wallis), and PlGF borderline non-significant ( $\mathrm{p}=0.064$, Kruskal-Wallis).

No correlations for PlGF or sFlt-1 with age and creatinine plasma levels were found.

\section{sFlt-1 and PIGF as prognostic markers}

Mean survival time was 91 months, in total 130 of 247 patients died. Survival analysis showed no statistically significant difference between patients below and above cut-off concentrations or median concentrations of sFlt-1 or PlGF. These results were confirmed by Cox regression analysis. This was true for both the whole cohort and each aetiological group in isolation (results not shown).

\section{Discussion}

VEGF signalling is known to be involved in vascular remodelling in PH [18].VEGF family members are overexpressed in lungs from $\mathrm{PH}$ patients and circulating VEGF is elevated in plasma of PH patients [8]. Inhibition of the VEGFR1 and VEGFR2 with the tyrosine kinase inhibitor SU5416 in combination with chronic hypoxia causes severe PAH in rats [19]. The role of VEGF signalling in PH development is not fully understood, but PlGF is thought to act pro-angiogenically by binding to Flt-1, whereas sFlt-1 can bind VEGF and thereby decrease its activity $[11,20]$.

In this study, sFlt-1 and PlGF plasma levels were measured in a population of 247 patients with $\mathrm{PH}$ of various aetiologies, and in 40 patients without $\mathrm{PH}$ who served as controls. Consecutive patients with suspicion of $\mathrm{PH}$ coming to the Giessen $\mathrm{PH}$ referral centre were included in the study. Right heart catheterisation was performed in every patient at baseline visit, in parallel with blood sample collection.

Plasma levels of sFlt-1 were significantly increased in patients with IPAH, APAH, CVD, and LD-PH compared with the non-PH controls, whereas PlGF levels were significantly increased in all $\mathrm{PH}$ groups. Use of PlGF for diagnosing PH resulted in a higher sensitivity than for sFlt-1, but sFlt-1 had an associated specificity of $100 \%$ for diagnosing $\mathrm{PH}$ in our patient cohort. When both parameters are combined the specificity still reaches $100 \%$ and sensitivity levels reach $62.4 \%$ for $\mathrm{PH}$ and $83.7 \%$ for $\mathrm{PAH}$. These data show a high potential for PlGF and sFlt-1 as diagnostic biomarkers for $\mathrm{PH}$.

The natriuretic peptides, BNP and its N-terminal fragment NT-proBNP, are established biomarkers for PH and are in routine use for the clinical management of patients with $\mathrm{PH}$. BNP levels are elevated in different groups of $\mathrm{PH}[4,21-23]$ and correlate with haemodynamic parameters [24], exercise capacity, WHO functional class [25] and are strong predictors of survival in PH [26, 27]. Also in direct comparison, BNP is a poor diagnostic tool in this study and should therefore be used differently than PlGF and sFlt-1. Whilst NT-proBNP and BNP can be used as prognostic biomarkers for patients with $\mathrm{PH}$, and can also mirror patients' therapeutic response, their plasma levels can also be normal in severely ill patients with $\mathrm{PH}$, and thus cannot be used to exclude a $\mathrm{PH}$ diagnosis.

It is important to emphasise that this is in contrast with the results of the current study, where PlGF and sFlt-1 levels show no correlation with $\mathrm{PH}$ disease severity; thus, PlGF and sFlt-1 have no prognostic potential. Nevertheless, PlGF and sFlt-1 seem likely to be useful diagnostic biomarkers for PH.

With their moderate sensitivity and their high specificity for PH (and PAH respectively), sFlt-1 and PlGF are reasonably suitable screening tools; however, their main strength lies in their almost unfailing specificity. Therefore, in the decision making tree of diagnostic workup of patients with suspected $\mathrm{PH}$, in cases of a negative test, confirmation by means of invasive haemodynamic assessment remains obligatory provided other non-invasive tests (e.g. echocardiography) remain suggestive of PH. In case of a positive test, sFlt- 1 and $\mathrm{PlGF}$ are novel tools that confirm PH with high certainty already at an early stage of diagnostic evaluation. It must be kept in mind, however, that right heart catheterisation remains mandatory for purposes of diagnostic classification and assessment of current haemodynamic severity of the disease. In addition, if the majority of non-invasive diagnostic tools are suggestive for a low probability of having $\mathrm{PH}$ during early work-up of symptomatic patients, but the novel biomarkers are positive, this in itself may become a novel means by which the likelihood to oversee $\mathrm{PH}$ in symptomatic patients is substantially reduced.

MaLHOTRA et al. [17] measured sFlt-1 serum levels in PAH patients, first degree IPAH and HPAH relatives and healthy controls. They found significantly increased sFlt-1 levels in PAH patients, with an AUC for diagnosing PAH that was comparable to this study. Furthermore, they found an association with increased NYHA and improved survival in patients below the median sFlt-1 level. We could confirm the association of sFlt-1 plasma levels with NYHA class in our study population, but we find no difference in survival time. Like in our study no correlation with haemodynamic parameters could be found. 
The missing correlation of sFlt-1 and PlGF with mean PAP, PVR, 6MWD and BNP suggests a pathological role that is not directly linked to the increased pressure in the pulmonary vasculature and the resulting changes of the right ventricle. Immunohistochemical stains of lungs from patients with $\mathrm{PAH}$ and from healthy donor lungs showed that the VEGF family members Flt-1 and PlGF are expressed in pulmonary arterial smooth muscle cells (see online supplement). As vascular remodelling in $\mathrm{PH}$ is mainly driven by smooth muscle cell proliferation, PlGF and Flt-1 may play a role in remodelling processes in PH. Moreover, as smooth muscle cells seem to be the main source of Flt-1 and PlGF in the PH lung, increased plasma levels of these molecules may mirror these changes in smooth muscle cells.

It is important to consider what pathophysiological mechanism might underlie the presence of PlGF and sFlt-1 as biomarkers for $\mathrm{PH}$, firstly by examining the documented role of these molecules in other conditions. Elevated sFlt-1 levels cause endothelial dysfunction during pre-eclampsia by binding circulating VEGF and PlGF, thereby preventing their interaction with endothelial cell-surface receptors. Levels of sFlt-1 are also elevated in sickle cell disease-PH compared with patients who have sickle cell disease without $\mathrm{PH}$, and a correlation with urinary albumin secretion was reported [15]. PlGF plasma levels are raised in sickle cell disease and higher levels associate with an increased incidence of vascular occlusive events [28].

Furthermore, in patients with peripheral and coronary artery disease, sFlt-1 plasma levels were lower than in healthy controls [16]. It is also notable that PlGF can be induced by HIF-1 $\alpha$ after exposure to hypoxia, both in vitro and in vivo [29-31].

In conclusion to this section, the relevance of sFlt-1 and PlGF plasma levels to specific pathophysiological mechanisms is unclear, but certainly warrants further research.

The control group in this study consists of patients visiting our clinic with symptoms similar to that in $\mathrm{PH}$ patients, and therefore underwent right heart catheterisation. This is an adequate control group to evaluate potential diagnostic biomarkers as a physician wants to differentiate patients with and without $\mathrm{PH}$ presenting with similar symptoms rather than to differentiate $\mathrm{PH}$ patients from healthy people. We included patients from different groups of the World $\mathrm{PH}$ classification to test the diagnostic potential of PlGF and sFlt-1 for PH versus non-PH in patients showing the same symptoms. In the literature plasma levels in healthy controls are reported to be $94.8 \mathrm{pg} \cdot \mathrm{mL}^{-1}$ for sFlt-1 and $13.7 \mathrm{pg} \cdot \mathrm{mL}^{-1}$ for PlGF [32]. Our control group shows higher levels for both biomarkers, but still significantly lower levels than $\mathrm{PH}$ patients.

Moreover, as plasma was only tested at the time of $\mathrm{PH}$ diagnosis, this may represent an early window into a mechanism that, conceivably, may only be present at this early stage. As such, this study showed a high potential for PlGF and sFlt-1 as diagnostic biomarkers for PH. Both proteins were expressed in remodelled vessels of patients with $\mathrm{PH}$, but increased plasma levels did not correlate with haemodynamic parameters, 6MWD and BNP.

This study focussed on the diagnostic potential of sFlt-1 and PlGF in PH patients, although the sensitivity was higher in PAH than for unselected $\mathrm{PH}$ patients. The decision, whether a patient should undergo invasive right heart catheterisation, is based on a variety of non-invasive measurements like echocardiography, imaging, clinical symptoms and circulating biomarkers, respectively. sFlt-1 and PlGF may become useful additional parameters which help to decide whether or not invasive assessment by means of right heart catheterisation is required.

Undoubtedly, further studies are necessary to understand the role of PlGF and sFlt-1 in PH, and greater numbers of patients may be needed to investigate correlations with disease parameters. We find comparable results to the study of MALHOTRA et al. [17], but confirmations in other patient cohorts are definitely needed. Furthermore, the investigation of patient cohorts over time is needed to reveal whether PlGF and sFlt-1 are only increased in the early phase of disease, and whether their plasma levels change in response to specific therapy.

\section{Acknowledgements}

We thank Ewa Bieniek (Universities of Giessen and Marburg Lung Center, Giessen, Germany) for technical assistance with histology. Richard Clark (Freelance Medical Writer, Dunchurch, Warwickshire, UK) and Claire Mulligan (Oxford PharmaGenesis Ltd, Oxford, UK) provided editorial support, funded by the University of Giessen.

This article contains parts of the results from the doctoral thesis of Marina dos Santos Guilherme.

\section{References}

1 Badesch DB, Champion HC, Sanchez MA, et al. Diagnosis and assessment of pulmonary arterial hypertension. J Am Coll Cardiol 2009; 54: S55-S66.

2 Stenmark KR, Meyrick B, Galie N, et al. Animal models of pulmonary arterial hypertension: The hope for etiological discovery and pharmacological cure. Am J Physiol Lung Cell Mol Physiol 2009; 297: L1013-L1032. 
3 Grünig E, Barner A, Bell M, et al. Nicht-invasive Diagnostik der pulmonalen Hypertonie: ESC/ERS-Leitlinien mit Kommentierung der Kolner Konsensus-Konferenz 2010 [Non-invasive diagnosis of pulmonary hypertension: ESC/ERS Guidelines with commentary of the Cologne Consensus Conference 2010]. Dtsch Med Wochenschr 2010; 135: Suppl. 3, S67-S77.

4 Nagaya N, Nishikimi T, Uematsu M, et al. Plasma brain natriuretic peptide as a prognostic indicator in patients with primary pulmonary hypertension. Circulation 2000; 102: 865-870.

5 Fujita M, Mason RJ, Cool C, et al. Pulmonary hypertension in TNF-alpha-overexpressing mice is associated with decreased vegf gene expression. J Appl Physiol 2002; 93: 2162-2170.

6 Forsythe JA, Jiang BH, Iyer NV, et al. Activation of vascular endothelial growth factor gene transcription by hypoxia-inducible factor 1. Mol Cell Biol 1996; 16: 4604-4613.

7 Tuder RM, Chacon M, Alger L, et al. Expression of angiogenesis-related molecules in plexiform lesions in severe pulmonary hypertension: Evidence for a process of disordered angiogenesis. J Pathol 2001; 195: 367-374.

8 Sundaram N, Tailor A, Mendelsohn L, et al. High levels of placenta growth factor in sickle cell disease promote pulmonary hypertension. Blood 2010; 116: 109-112.

9 Wu FT, Stefanini MO, Mac Gabhann F, et al. A systems biology perspective on SVEGFR1: Its biological function, pathogenic role and therapeutic use. J Cell Mol Med 2010; 14: 528-552.

10 Park JE, Chen HH, Winer J, et al. Placenta growth factor. Potentiation of vascular endothelial growth factor bioactivity, in vitro and in vivo, and high affinity binding to Flt-1 but not to flk-1/kdr. J Biol Chem 1994; 269 : 25646-25654.

11 Autiero M, Waltenberger J, Communi D, et al. Role of PLGF in the intra- and intermolecular cross talk between the VEGF receptors flt1 and flk1. Nat Med 2003; 9: 936-943.

12 Tarallo V, Vesci L, Capasso O, et al. A placental growth factor variant unable to recognize vascular endothelial growth factor (VEGF) receptor-1 inhibits VEGF-dependent tumor angiogenesis via heterodimerization. Cancer Res 2010; 70: 1804-1813.

13 Levine RJ, Maynard SE, Qian C, et al. Circulating angiogenic factors and the risk of preeclampsia. N Engl J Med 2004; 350: 672-683.

14 Maynard SE, Min JY, Merchan J, et al. Excess placental soluble fms-like tyrosine kinase 1 (sflt1) may contribute to endothelial dysfunction, hypertension, and proteinuria in preeclampsia. J Clin Invest 2003; 111: 649-658.

15 Ataga KI, Brittain JE, Jones SK, et al. Association of soluble fms-like tyrosine kinase-1 with pulmonary hypertension and haemolysis in sickle cell disease. Br J Haematol 2010; 152: 485-491.

16 Blann AD, Belgore FM, McCollum CN, et al. Vascular endothelial growth factor and its receptor, flt-1, in the plasma of patients with coronary or peripheral atherosclerosis, or type II diabetes. Clin Sci (Lond) 2002; 102: 187-194.

17 Malhotra R, Paskin-Flerlage S, Zamanian RT, et al. Circulating angiogenic modulatory factors predict survival and functional class in pulmonary arterial hypertension. Pulm Circ 2013; 3: 369-380.

18 Voelkel NF, Gomez-Arroyo J. The role of vascular endothelial growth factor in pulmonary arterial hypertension: The angiogenesis paradox. Am J Respir Cell Mol Biol 2014; 51: 474-484.

19 Taraseviciene-Stewart L, Kasahara Y, Alger L, et al. Inhibition of the VEGF receptor 2 combined with chronic hypoxia causes cell death-dependent pulmonary endothelial cell proliferation and severe pulmonary hypertension. FASEB J 2001; 15: 427-438

20 Kendall RL, Thomas KA. Inhibition of vascular endothelial cell growth factor activity by an endogenously encoded soluble receptor. Proc Natl Acad Sci USA 1993; 90: 10705-10709.

21 Bozkanat E, Tozkoparan E, Baysan O, et al. The significance of elevated brain natriuretic peptide levels in chronic obstructive pulmonary disease. I Int Med Res 2005; 33: 537-544

22 Leuchte $\mathrm{HH}$, Neurohr C, Baumgartner R, et al. Brain natriuretic peptide and exercise capacity in lung fibrosis and pulmonary hypertension. Am J Respir Crit Care Med 2004; 170: 360-365.

23 Nagaya N, Nishikimi T, Uematsu M, et al. Secretion patterns of brain natriuretic peptide and atrial natriuretic peptide in patients with or without pulmonary hypertension complicating atrial septal defect. Am Heart J 1998; 136: $297-301$.

24 Leuchte HH, Holzapfel M, Baumgartner RA, et al. Characterization of brain natriuretic peptide in long-term follow-up of pulmonary arterial hypertension. Chest 2005; 128: 2368-2374.

25 Leuchte $\mathrm{HH}$, Holzapfel M, Baumgartner RA, et al. Clinical significance of brain natriuretic peptide in primary pulmonary hypertension. J Am Coll Cardiol 2004; 43: 764-770.

26 Park MH, Scott RL, Uber PA, et al. Usefulness of b-type natriuretic peptide as a predictor of treatment outcome in pulmonary arterial hypertension. Congest Heart Fail 2004; 10: 221-225.

27 Fijalkowska A, Kurzyna M, Torbicki A, et al. Serum n-terminal brain natriuretic peptide as a prognostic parameter in patients with pulmonary hypertension. Chest 2006; 129: 1313-1321.

28 Perelman N, Selvaraj SK, Batra S, et al. Placenta growth factor activates monocytes and correlates with sickle cell disease severity. Blood 2003; 102: 1506-1514.

29 Kelly BD, Hackett SF, Hirota K, et al. Cell type-specific regulation of angiogenic growth factor gene expression and induction of angiogenesis in nonischemic tissue by a constitutively active form of hypoxia-inducible factor 1 . Circ Res 2003; 93: 1074-1081.

30 Ma C, Wang Y, Shen T, et al. Placenta growth factor mediates angiogenesis in hypoxic pulmonary hypertension. Prostaglandins Leukot Essent Fatty Acids 2013; 89: 159-168.

31 Yamakawa M, Liu LX, Date T, et al. Hypoxia-inducible factor-1 mediates activation of cultured vascular endothelial cells by inducing multiple angiogenic factors. Circ Res 2003; 93: 664-673.

32 Trelinski J, Wierzbowska A, Krawczynska A, et al. Circulating endothelial cells in essential thrombocythemia and polycythemia vera: Correlation with jak2-v617f mutational status, angiogenic factors and coagulation activation markers. Int J Hematol 2010; 91: 792-798. 